\section{Analysis of Fusarium ear rot and fumonisin contamination in testcrosses of a maize biparental population}

\section{Vlatko Galići ${ }^{*}$, Domagoj Šimić ${ }^{1}$, Mario Franić ${ }^{2}$, Andrija Brkićc ${ }^{1}$, Antun Jambrović ${ }^{1}$, Josip Brkić ${ }^{1}$ and Tatjana Ledenčan ${ }^{1}$}

\begin{abstract}
Fusarium ear rot (FER) negatively affects maize production worldwide and poses a serious threat to human health for producing mycotoxins. The objectives of our study were to assess the factors affecting FER and fumonisin contamination in testcrosses of a maize population. In the trials, seeds of 191 testcrosses of IBMSyn4, 11 checks and 2 replications of parental lines (a total of 216 hybrids) were sown in three environments, in 2014 and 2015. FER disease intensity (DI), fumonisin contamination (FUMc) and number of ears with signs of European corn borer (ECB) attack were measured. Strong phenotypic and weak genetic correlations between DI, FUMc and ECB indicate randomness in the interaction of those traits. We detected three QTLs (chr.1, 2 and 6) for DI (LOD scores 3.77-5.06). The QTL on chr.2, confirmed across the environments, can serve as a guideline in breeding for FER resistance.
\end{abstract}

Keywords: QTL mapping, maize genetic resistance, Fusarium ear rot.

\section{INTRODUCTION}

Diseases caused by Fusarium spp. fungi negatively affect maize production worldwide. As is generally known, Fusarium ear rot (FER) reduces the maize grain yield (Nagy et al. 2011), and the main causal agents Fusarium verticillioides and Fusarium proliferatum produce mycotoxins of the fumonisin group that pose a serious threat to human and animal health (Scott 2012). An increasing awareness of the deleterious effects of fumonisin on human health has led to the recommendation of legislative regulation by food security agencies in US, Europe and Brazil (Taylor 2001, Kyprianou 2006a, Mouthino 2011). The upper limits of the total fumonisin content in regulations vary between 1 and $2 \mathrm{mg} /$ $\mathrm{kg}$ for corn for human food consumption.

Although there are numerous factors affecting fungal growth, FER severity seems to be largely determined by insect attacks (Bakan et al. 2002) and favorable environmental conditions. The insect European corn borer (Ostrinia nubilalis Hübner, ECB) represents an important vector for FER development in Europe, due to its feeding activities (Alma et al. 2005), and it also increases the incidence of fumonisin contamination (Mazzoni et al. 2011). Agricultural practices, e.g., a high planting density, are additional factors leading to higher FER disease intensity by modulating the microclimatic conditions, making them favorable for fungi development (Blandino et al. 2008). Even in case of high FER severity, fumonisin contamination sometimes occurs without symptoms (Munkvold et
Crop Breeding and Applied Biotechnology 19: 40-46, 2019 Brazilian Society of Plant Breeding. Printed in Brazil http://dx.doi.org/10.1590/198470332019v19n1a06

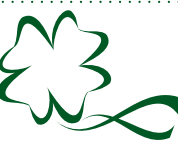

70332019v19n1a06 
al. 1997), because its production is influenced by many physico-chemical, environmental and agronomical conditions (Samapundo et al. 2005, Herrera et al. 2010).

As agronomic control methods of fumonisin contamination often fail, and there is a considerable genetic variability for FER resistance in maize germplasm (Santiago et al. 2013, Schwantes et al. 2018), genetic improvement might be the optimal strategy in future maize breeding for tolerable fumonisin contamination in food and feed (Maschietto et al. 2017). To date, many QTL studies were conducted in populations with low-density genetic maps, and QTL studies in densely mapped populations are rare (Lanubile et al. 2017). The relative efficiency of selection for FER resistance is greater in testcrosses than in inbred lines, and due to mainly additive gene action, the general combining ability of the inbreds is more important than the specific combining ability (Löffel et al. 2011, Hung and Holland 2012). The resemblance between the testcross performances with two testers appears to be high, so the use of a single tester should suffice for the evaluation of material for FER resistance (Löffel et al. 2011) provided it is heterotic to the tester. Phenotyping a high number of progenies for FUMc is costly, as the protocols require the use of expensive measuring equipment and immunoassays. Due to the moderate phenotypic and high genetic correlations between FER and FUMc, selection for lower FER should be able to frequently identify lines with lower FUMc (Robertson et al. 2006). Selection for resistance to FER can be performed in environments where disease pressure is severe enough to cause consistent infection and visible symptoms (Duvick 2001).

In our study, the ECB attack was considered an environmental factor controlling FER. The objective of the current study was to investigate the environmental and genetic factors affecting FER resistance and fumonisin contamination in testcrosses of a maize biparental population.

\section{MATERIAL AND METHODS}

\section{Experimental design}

The experiments were carried out on experimental fields of the Agricultural Institute Osijek, in Osijek, Croatia, in 2014 and 2015. The 191 recombinant inbred lines of IBMsyn4 (Intermated B73xMo17, Lee et al. 2002) population were topcrossed with a tester line of the Agricultural Institute Osijek, 84-28A with lodent background (moderately susceptible to FER) that combines well with both IBM parents to produce testcrosses. Along with population testcrosses, two replications of testcrosses of each population parental line (B73 and Mo17), along with 11 checks were sown. A total of 216 hybrids were tested. The experiment was arranged in a rectangular lattice without replications, with 27 plots per block, in 8 blocks in both years. Two trials with different planting densities in 2014 of $\sim 56000$ and 95000 plants/ ha (OS14N and OS14H) were considered separate environments along with a single trial in 2015 with 56000 plants/ ha (OS15). Each two-row plot covered $8.4 \mathrm{~m}^{2}$ and contained 47 plants at normal plant density and 80 plants at high plant density. In 2014, sowing was performed on April 23, and in 2015, on April 26. In both years, sowing on plots was performed after soybean as preceding crop in a three-year crop rotation (soybean, maize, wheat/barley). Standard agrotechniques were performed, with spraying of pre- emergence herbicide, a corrective spray in stage V9, and a single soil dressing with calcium ammonium nitrate was applied simultaneously with cultural practices in stage V10. Plots were hand-harvested in October and the plants subjected to phytopathological assessment. Meteorological data for both study years were obtained from the Croatian Meteorological and Hydrological service. Vapor pressure deficit (VPD) was calculated according to Allen et al. (1998), based on saturation vapor pressure, air temperature and humidity.

\section{Assessment of Fusarium ear rot and fuminsin contamination}

An environment with high ECB incidence of was chosen, as this insect is one of the major Fusarium vectors. The plots were visually rated for number of plants showing clear signs of ECB attack on ears (holes and tunnels) and were expressed as percentages. The disease intensity (DI) of FER was rated visually according to a method developed by Reid and Zhu (2005), and further simplified by Santiago et al. (2013). Briefly, husks were removed from all harvested ears and they were rated for FER on a seven-point scale ( $1=$ no visible symptoms, $2=1-3 \%, 3=4-10 \%, 4=11-25 \%, 5=$ $26-50 \%, 6=51-75 \%$ and $7=>75 \%)$. Visual ratings were expressed as percentages of points according to the proposal of Reid and Zhu (2005) to reduce the pooling error introduced by wide grading. Results for all ears on a single plot were averaged. All ears of a plot were husked, and grain sampling for fumonisin contamination assessment was performed 
according to the European Commission Regulation No 401/2006 (Kyprianou 2006b). The total fumonisin content (FUMc) was measured with ROSA (Rapid One-Step Assay) technology using a lateral flow test kit (Charm Sciences INC, USA), a method shown to be closely correlated to high performance liquid chromatography (HPLC) (Chilaka et al. 2013). A $50 \mathrm{~g}$ ground grain sample was mixed with $100 \mathrm{~mL} \mathrm{70 \%} \mathrm{methanol,} \mathrm{shaken} \mathrm{for} 2 \mathrm{~min}$, and filtered. The sample extracts were diluted two or three times with dilution buffer to adjust the fumonisin content for the measuring range of the device and pipetted onto measuring strips. The fumonisin contents in samples were expressed as $\mathrm{mg} / \mathrm{kg}$.

\section{Genotyping data}

The maize IBMSyn4 population was genotyped by various methods (Andorf et al. 2010). We used the IBM Neighbors 2 linkage map anchored with 2178 genetically mapped markers (mostly SNP and SSR). The total map length was 7090 $\mathrm{CM}$ and the average distance between markers $3.2 \mathrm{cM}$. As the IBM population consists of recombinant inbred lines developed over four successive generations of intermating (recombination), the centiMorgan value of the IBM population is actually IBM centiMorgan, as there was an increase in both map size and density (Lee et al. 2002, Falque 2005). The QTL results reported here are in IBM cM (1 IBM cM 4 cM in F2) on the IBM2 Neighbor map.

\section{QTL analysis}

For the QTL analyses, QTL IciMapping software, version 4.1 was used (Meng et al. 2015), in a twofold approach. First, stepwise regression and model selection were run to select the optimal mapping procedure and covariates for further analysis. The probability of inclusion of the QTL in stepwise regression was set at $p=0.001$. Next, inclusive composite interval mapping (ICIM) was performed at a mapping step size of $1 \mathrm{cM}$ and 1000 permutations, to determine the statistical significance threshold. The calculated threshold based on 1000 permutation test was 3.62. The peak LOD score was considered the QTL position, and confidence intervals of detected QTL were determined based on a unit drop in LOD score. To investigate the QTL x environment interaction, we performed the ICIM-MET (multi-environment trial) analysis as implemented in QTL IciMapping software. The calculated LOD threshold based on 1000 permutations, indicating significance of the QTL in three environments, was 5.07.

\section{Statistical analysis}

Phenotypic correlation coefficients among traits were calculated using Spearman's rank correlation test. Genetic correlations were assessed from the genetic covariance matrix based on the mixed linear model approach in $\mathrm{R}$ package sommer (Covarrubias-Pazaran 2016). Variance components were estimated in the R Ime4 package (Bates et al. 2015). Genotype, environment, density and genotype $x$ density interactions were considered random, while the block effects were treated as fixed. Entry-mean heritabilities were estimated based on a formula modified from that of Hallauer et al. (2010):

$$
H^{2}=\frac{\hat{\sigma}_{G}^{2}}{\hat{\sigma}_{G}^{2}+\frac{\hat{\sigma}_{G D}^{2}}{d}+\frac{\hat{\sigma}_{e}^{2}}{d e}}
$$

where $\hat{\sigma}_{G}^{2}$ stands for variance due to genotype; $\hat{\sigma}_{G D}^{2}$ for variance due to the genotype $\times$ density interaction; $\hat{\sigma}_{e}^{2}$ is error variance and $d$ and $e$ are the numbers of densities and environments, respectively.

\section{RESULTS AND DISCUSSION}

Significantly higher mean values for DI, FUMc, and ECB were observed between all three environments (Figure 1). On a total of $94 \%$ of the plots FER symptoms were observed. Mean values of FUMc in all three environments exceeded the threshold for direct human consumption $\left(1 \mathrm{mg} \mathrm{kg}^{-1}\right)$. Of all analyzed samples, $71 \%$ had detectable levels of fumonisin and $44 \%$ exceeded threshold levels for use in milling $\left(2 \mathrm{mg} \mathrm{kg}^{-1}\right)$. Of the 287 samples exceeding the tolerable amount of fumonisin in foodstuff, $39 \%$ belonged to environment OS14N, $50 \%$ to OS14H and $11 \%$ to OS15.

The highest DI and FUMc were determined in environments OS14N and OS14H with lowest VPD, and lowest average temperatures in July and August (Table 1) within the favorable range for fumonisin production (Samapundo et al. 2005). The traits FER and FUMc are strongly influenced by water activity, a physical property of water solutions, and 
Table 1. Average temperatures $\left(\mathrm{t}\left({ }^{\circ} \mathrm{C}\right) \pm\right.$ S.E.), precipitation and vapor pressure deficit (VPD \pm S.E.) in July and August 2014 and 2015

\begin{tabular}{|c|c|c|c|c|c|c|}
\hline \multirow{2}{*}{ Year } & \multicolumn{2}{|c|}{$\mathrm{t}\left({ }^{\circ} \mathrm{C}\right)$} & \multicolumn{2}{|c|}{ Precipitation (mm) } & \multicolumn{2}{|c|}{ VPD (Pa) } \\
\hline & July & August & July & August & July & August \\
\hline 2014 & $22.3 \pm 2.1$ & $21.1 \pm 2.5$ & 82.6 & 92.5 & $807 \pm 237$ & $709 \pm 219$ \\
\hline 2015 & $24.6 \pm 3.2$ & $24.5 \pm 3.6$ & 24.9 & 38.9 & $1272 \pm 402$ & $1320 \pm 578$ \\
\hline
\end{tabular}
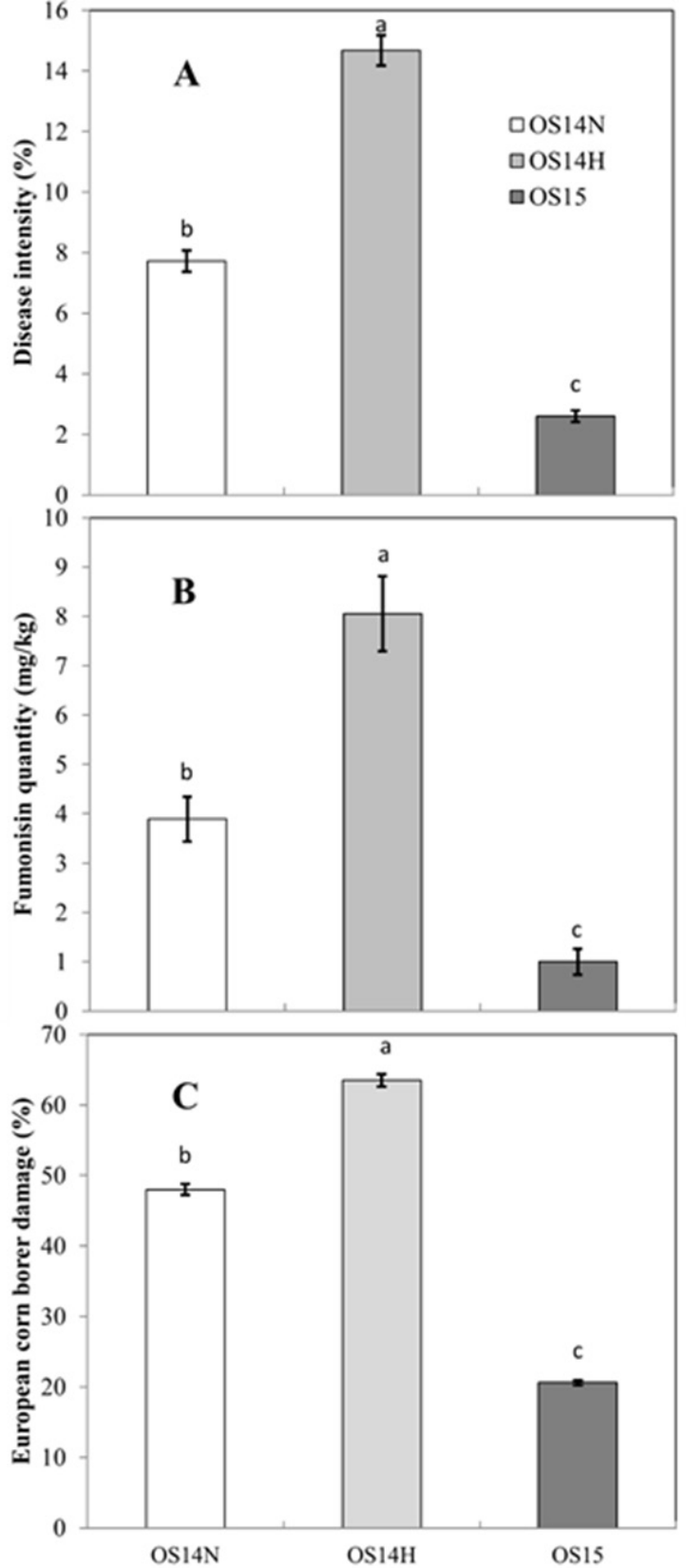

Figure 1. Means and standard errors of FER disease intensity (A), Fumonisin contamination (B) and damage caused by the insect ECB (C). Different letters represent statistical significance at $\alpha=0.05$. air temperature (Samapundo et al. 2005). A water activity $\left(a_{w}\right)$ of 1 represents the osmotic state of distilled water, and growth of Fusarium colonies appears to be the fastest at air temperatures around $30^{\circ} \mathrm{C}$ and $a_{w}$ close to 1 (0.969). The same conditions also seem to boost fumonisin production, although fumonisin production rates were found to be similarly high at the same $a_{w}$ level, when temperatures were $22{ }^{\circ} \mathrm{C}$ and $25^{\circ} \mathrm{C}$. Lower values of $a_{w}$ were found to negatively affect fumonisin production. Parameter $a_{w}$ is a property of water solutions closely related to VPD, and lower values of $a_{w}$ correspond to higher levels of VPD (Kiyosawa 2003). The mean FUMc in environment OS14H was higher than the tolerable fumonisin levels in grains intended for processing $\left(4 \mathrm{mg} \mathrm{kg}^{-1}\right)$ or as feed for pigs and horses $(5 \mathrm{mg}$ $\mathrm{kg}^{-1}$ ) (Montagana et al. 2012).

Higher plant densities are known to increase DI and FUMc, mostly through induction of changes in microclimate within the crop that favor pathogen growth and development (Blandino et al. 2008). Furthermore, temperate humid conditions also favor a faster development and higher incidence of ECB (Löffler et al. 2005), an insect closely associated with FER (Alma et al. 2005) and FUMc (Mazzoni et al. 2011). Moderate to strong significant phenotypic correlations of ECB with DI and FUMc were also observed in the current study (Table 3). High incidence of ECB (20.6 $-63.5 \%$, Table 2 ) was the main reason for high levels of DI and FUMc in all three environments. Low heritabilities were obtained for all measured traits (Table 2). The first factor causing low heritability of FUMc was randomness present in FER induction through random factor ECB. The genetic correlation of DI and FUMc was not significant, while the phenotypic correlation was high and significant (Table 3). The reason for this downward bias of genetic correlation compared to phenotypic correlations was the small estimate of genetic variance for FUMc, which consequently led to the lower genetic covariance estimates (Hallauer et al. 2010). The FER inoculation with spore suspension is known to increase the genetic component of variance for the traits DI and FUMc (Reid and Zhu 2005) and hence the heritability, as the time of infection is the same for all entries. Disease induction by the environmental factor ECB lowered the genetic component of variance for FUMc in our study, as the time and thus the conditions of infection were not 
the same for all tested hybrids. The experimental design, in which none of the experiments was replicated, was the second factor, which inflated the error variance in heritability calculation (Hallauer et al. 2010), although unreplicated trials with replicated checks can be effective if the number of genotypes is kept constant (Bernardo 2010). Due to the very low estimate of genetic variance component that induced the low heritability estimate, FUMc was excluded from the QTL analysis. FUMc analyses are expensive and symptomatic kernels are expected to have higher levels of fumonisin due to moderate, significant positive correlations. In addition, indirect selection for FER resistance is expected to result in lower frequency of high FUMc concentrations in selected progenies (Clements et al. 2004, Robertson et al. 2006). Mo17 showed comparable but $0.35 \%$ lower $\mathrm{DI}$ as well as $0.85 \mathrm{mg} \mathrm{kg}^{-1}$ lower fumonisin contamination compared to $B 73$, although none of the differences was significant (Table 2). Santiago et al. (2013) showed that Mo17, the male parent of maize IBM population accumulates less fumonisin and has a $0.38 \%$ higher DI compared to the IBM population female parent, B73. The differences between studies can be attributed to the different agroecological conditions between Spain and Croatia.

We mapped loci with significant phenotypic effects on DI on chromosomes 1, 2 and 6 (Table 4). Maschietto et al. (2017) confirmed two detected QTL (chromosomes 1 and 2), and the locus with highest LOD score observed in our study (5.06) in bin 2.04 was also found in their study, controlling for both DI and FUMc. The mentioned study was conducted with artificial infection, controlling for high DI rate and the time of pathogen occurrence. The peak position of QTL on chromosome 1 was mapped directly at the position where gene bh/h43 is mapped. bh/h43 codes for basic helix-loop-helix transcription factors included in pathways regulating response to biotic stress in many plant species (Feller et al. 2011). QTL on chromosome 2 was mapped in the region where cta1 gene is found, coding for enzyme chitinase-A1 known for induction of resistance to various fungi in plants (Noonan et al. 2017). Chynolytic enzymes are found in microorganisms, plants and animals, and their function is the hydrolytic degradation of chitin, a component of fungal cell wall. In their study, Hawkins et al. (2015) mapped the QTL for Aspergilus flavus resistance in the same region in two maize populations, where the QTL explained $24 \%$ and $9 \%$, respectively, of the total phenotypic variation for resistance. The authors identified three genes that can confer resistance in this region, only $30 \mathrm{~kb}$ apart, so the cta1 might not be the sole causative agent for this QTL, due to linkage. Also, the QTL on chromosome 2 was mapped in close proximity (6 IBM cM upstream) to gene nactf36, coding for NAC transcription factor. Transcription factors of the NAC family play one of the most important roles in plant biotic and abiotic stress responses and host recognition of pathogens (Nuruzzaman et al. 2013). The QTL on chromosome 2 was further confirmed in MET analysis, having a significant phenotypic effect in all three environments (Table 4). Additive effects were negative in environments with normal plant density, while the sign of effect changed in high plant density environments. Complex regulatory responses are expected from transcription factors mediating plant response to many stresses.

The genetic factors affecting the FER tolerance found in our study can be used as valuable guidelines in breeding programs targeting improved hybrid FER tolerance since they were mapped in a densely genotyped maize population, and studies of genetic factors affecting FER are rare in densely mapped populations (Lanubile et al. 2017). The experimental 
Analysis of Fusarium ear rot and fumonisin contamination in testcrosses of a maize biparental population

Table 4. Results of inclusive composite interval mapping (ICIM) for FER disease intensity (DI) and the results of ICIM-MET analysis for FER disease intensity (DI) (Combined)

\begin{tabular}{|c|c|c|c|c|c|c|c|c|c|c|}
\hline Environment & Bin $^{a}$ & Position (cM) & Interval $(\mathrm{cM})^{\mathrm{b}}$ & LOD & $R^{2}(\%)^{c}$ & $R^{2}(\text { Add })^{\mathrm{d}}$ & $\boldsymbol{R}^{2}(\mathrm{AxE})^{\mathrm{e}}$ & AxEO1 $^{f}$ & AxE02 & AxE03 \\
\hline OS14N & $6.02 / 03$ & 159 & $154.5-162.5$ & 4.16 & 10.03 & - & - & 1.51 & - & - \\
\hline OS14H & 2.04 & 250 & $248.5-251.5$ & 5.06 & 10.03 & - & - & - & 2.28 & - \\
\hline OS15 & 1.09 & 815 & $813.5-817.5$ & 3.77 & 6.02 & - & - & - & - & -0.75 \\
\hline Combined & 2 & 250 & $248.5-251.5$ & 5.96 & 13.59 & 7.27 & 6.32 & -0.33 & 1.27 & -0.94 \\
\hline
\end{tabular}

${ }^{\text {a }}$ Bin represents linkage group followed by region after decimal

${ }^{b}$ One LOD drop interval in cM

c Phenotypic variance explained by the QTL

${ }^{d}$ Phenotypic variance explained by the additive QTL effects

e Phenotypic variance explained by the QTL x environment interaction

${ }^{f}$ Additive effects of QTL x environment interactions

design used in the current study did not include artificial inoculation, which may have been the reason for the low or no genetic correlations between DI and fumonisin contamination, although moderate to strong phenotypic correlations indicate that lowering the DI should consequently result in lower FUMc. Breeding for lower DI would thus represent a feasible and more cost-effective approach in breeding for lower FUMc, as phenotyping for this trait in many progenies is expensive. The use of artificial inoculation is advised in environments with low incidence of ECB to increase the power of selection for lower DI and consequently FUMc.

\section{ACKNOWLEDGEMENTS}

This research was funded by the Croatian Science Foundation project 5707: "Genetics and physiology of multiple stress tolerance in maize".

\section{REFERENCES}

Allen RG, Pereira LS, Raes D and Smith M (1998) Crop evapotranspiration - guidelines for computing crop water requirements. FAO Irrigation and Drainage Paper 56. FAO, Rome, 15p.

Alma A, Lessio F, Reyneri A and Blandino M (2005) Relationships between Ostrinia nubilalis (Lepidoptera: Crambidae) feeding activity, crop technique and mycotoxin contamination of corn kernel in northwestern Italy. International Journal of Pest Management 51: 165-173.

Andorf CM, Lawrence CJ, Harper LC, Schaeffer ML, Campbell DA and Sen TZ (2010) The Locus Lookup tool at MaizeGDB: identification of genomic regions in maize by integrating sequence information with physical and genetic maps. Bioinformatics 26: 434-436.

Bakan B, Melcion D, Richard-Molard D and Chagnier B (2002) Fungal growth and fusarium mycotoxin content in isogenic traditional maize and genetically modified maize grown in france and spain. Journal of Agricultural and Food Chemistry 50: 728-731.

Bates D, Mächler M, Bolker BM and Walker SC (2015) Fitting linear mixedeffects models using Ime4. Journal of Statistical Software 67: 1-48.

Bernardo R (2010) Breeding for quantitative traits in plants. Stemma Press, Minnesota, 390p.

Blandino M, Reyneri A and Vanara F (2008) Effect of plant density on toxigenic fungal infection and mycotoxin contamination of maize kernels. Field Crops Research 106: 234-241.
Chilaka CA, De Kock S and Dutton MF (2013) Comparative profiling of different analytical methods for fumonisin detection in maize. Journal of Microbiology, Biotechnology and Food Sciences 2: 833-849.

Clements MJ, Maragos CM, Pataky JK and White DG (2004) Sources of resistance to fumonisin accumulation in grain and fusarium ear and kernel rot of corn. Phytopathology 94: 251-260.

Covarrubias-Pazaran G (2016) Genome-assisted prediction of quantitative traits using the $r$ package sommer. PLoS One 11: e0156744.

Duvick J (2001) Prospects for reducing fumonisin contamination of maize through genetic modification. Environmental Health Perspectives 109: 337-342.

Falque M, Décousset L, Dervins D, Jacob AM, Joets J, Martinant JP, Raffoux X, Ribière N, Ridel C, Samson D, Charcosset A and Murigneux A (2005) Linkage mapping of 1454 new maize candidate gene Loci. Genetics 170: $1957-1966$

Feller A, Machemer K, Braun EL and Grotewold E (2011) Evolutionary and comparative analysis of MYB and bHLH plant transcription factors. The Plant Journal 66: 94-116.

Hallauer AR, Carena MJ and Miranda-Filho JB (2010) Quantitative genetics in maize breeding. Springer, New York, 664p.

Hawkins LK, Mylroie JE, Oliveira DA, Smith JS Ozkan S, Windham GL, Williams WP and Warburton ML (2015) Characterization of the maize chitinase genes and their effect on Aspergillus flavus and aflatoxin accumulation resistance. PLoS ONE 10: e0126185. 


\section{$\checkmark$ Galić et al.}

Herrera M, Conchello P, Juan T, Estopañan G, Herrera A and Ariño A (2010) Fumonisin concentrations in maize as affected by physico-chemical, environmental and agronomical conditions. Maydica 55: 121-126.

Hung HY and Holland JB (2012) Diallel Analysis of resistance to Fusarium ear rot and fumonisin contamination in maize. Crop Science 52: 2173-2181.

Kiyosawa K (2003) Theoretical and experimental studies on freezing point depression and vapor pressure deficit as methods to measure osmotic pressure of aqueous polyethylene glycol and bovine serum albumin solutions. Biophysical Chemistry 104: 171-188.

Kyprianou M (2006a) December 2006 setting maximum levels for certain contaminants in foodstuffs. Official Journal of the European Union L 364: 5-24.

Kyprianou M (2006b) Laying down the methods of sampling and analysis for the official control of the levels of mycotoxins in foodstuffs. Official Journal of the European Union L 70: 12-34.

Lanubile A, Maschietto V, Borelli VM, Stagnati L, Logrieco AF and Marocco A (2017) Molecular basis of resistance to fusarium ear rot in maize. Frontiers in Plant Science 8: 1774.

Lee M, Sharopova N, Beavis N, Grant WD, Katt M, Blair D and Hallauer $A R$ (2002) Expanding the genetic map of maize with the intermated B73 × Mo17 (IBM) population. Plant Molecular Biology 48: 453-461.

Löffel CM, Kessel B, Ouzunova M and Meidaner T (2011) Covariation between line and testcross performance for reduced mycotoxin concentrations in European maize after silk channel inoculation of two Fusarium species. Theoretical and Applied Genetics 122: 925-934.

Löffler CM, Wei J, Fast T, Gogerty J, Langton S, Bergman M, Merrill B and Cooper M (2005) Classification of maize environments using crop simulation and geographic information systems. Crop Science 45: 1708-1716.

Maschietto V, Colombi C, Pirona R, Pea G, Strozzi F, Marocco A, Rossini L and Lanubile A (2017) QTL mapping and candidate genes for resistance to Fusarium ear rot and fumonisin contamination in maize. BMC Plant Biology 17: 20.

Mazzoni E, Scandolara A, Giorni P, Pietri A and Battilani P (2011) Field control of Fusarium ear rot, Ostrinia nubilalis (Hubner), and fumonisin in maize kernels. Pest Management Science 67: 458-465.

Meng L, Li H, Zhang L and Wang J (2015) QTL IciMapping: Integrated software for genetic linkage map construction and quantitative trait locus mapping in biparental populations. The Crop Journal 3: 269-283.
Montagana MT, De Giglio O and Napoli C (2012) Mycotoxins in foodstuffs: Italian regulations in the European framework. Annali Di Igiene 24: 475-489.

Mouthino JC (2011) Dispõe sobre limites máximos tolerados (LMT) para micotoxinas em alimentos. Ministério da Saúde, Agência Nacional de Vigilância Sanitária, Brasília, 3p. (RDC 7).

Munkvold GP, Hellmich RL and Showers WB (1997) Reduced Fusarium ear rot and symptomless infection in kernels of maize genetically engineered for European corn borer resistance. Disease Control and Pest Management 87: 1071-1077.

Nagy E, Haş V, Haş I, Suciu A and Florian V (2011) The influence of fusarium ear infection on the maize yield and mycotoxin content (TransylvaniaRomania). Plant Breeding and Seed Science 64: 35-44.

Noonan J, Williams WP and Shan X (2017) Investigation of antimicrobial peptide genes associated with fungus and insect resistance in maize. International Journal of Molecular Sciences 18: 1938.

Nuruzzaman M, Sharoni AM and Kikuchi S (2013) Roles of NAC transcription factors in the regulation of biotic and abiotic stress responses in plants. Frontiers in Microbiology 4: 248.

Reid LM and Zhu X (2005) Screening corn for resistance to common diseases in Canada. Agriculture and Agri-Food Canada, Ottawa, 29p.

Robertson LA, Kleinschmidt CE, White DG, Payne GA, Maragos CM and Holland JB (2006) Heritabilities and correlations of Fusarium ear rot resistance and fumonisin contamination resistance in two maize populations. Crop Science 46: 353-361.

Samapundo S, Devlighere F, De Mulenaer B and Debevere J (2005) Effect of water activity and temperature on growth and the relationship between fumonisin production and the radial growth of Fusarium verticillioides and Fusarium proliferatum on corn. Journal of Food Protection 68: 1054-1059.

Santiago R, Cao A, Malvar RA, Reid LM and Butrón A (2013) Assessment of corn resistance to fumonisin accumulation in a broad collection of inbred lines. Field Crops Research 149: 193-202.

Schwantes IA, Amaral Júnior AT, Vivas M, Almeida Filho JE, Kamphorst H, Guimarães AG and Khan S (2018) Inheritance of resistance to Fusarium ear rot in popcorn. Crop Breeding and Applied Biotechnology 18: 81-88.

Scott PM (2012) Recent research on fumonisin: a review. Food Additives and Contaminants 29: 242-248.

Traylor S (2001) Guidance for industry: fumonisin levels in human foods and animal feeds. Food \& Drug Administration, Rockville, $4 p$.

(cc) EY This is an Open Access article distributed under the terms of the Creative Commons Attribution License, which permits unrestricted use, distribution, and reproduction in any medium, provided the original work is properly cited. 